\title{
Variations in Tomotherapy Beam Outputs: A Multiple-Institutional Investigation
}

\author{
Hidetoshi Shimizu1, Koji Sasaki ${ }^{2}$, Kentaro Sugi ${ }^{3}$, Hiroshi Fukuma ${ }^{4}$, Tadashi Nakabayashi ${ }^{5}$, \\ Taiki Isomura', Hiroyuki Tachibana ${ }^{1}$, Takeshi Kodaira ${ }^{1}$
}

${ }^{1}$ Department of Radiation Oncology, Aichi Cancer Center Hospital, Aichi, Japan

${ }^{2}$ Department of Radiation Therapy Education and Research, Graduate School of Radiological Technology, Gunma Prefectural

College of Health Sciences, Gunma, Japan

${ }^{3}$ Hitachi, Ltd. Healthcare Business Unit, Tokyo, Japan

${ }^{4}$ Department of Radiology, Nagoya City University Hospital, Aichi, Japan

${ }^{5}$ Customer Support \& Area Operation Headquarters, Physics \& Clinical Support, Accuray Japan K.K., Tokyo, Japan

Email: hishimizu@aichi-cc.jp

\begin{abstract}
How to cite this paper: Shimizu, H., Sasaki, K., Sugi, K., Fukuma, H., Nakabayashi, T., Isomura, T., Tachibana, H. and Kodaira, T. (2018) Variations in Tomotherapy Beam Outputs: A Multiple-Institutional Investigation. International Journal of Medical Physics, Clinical Engineering and Radiation Oncology, 7, 87-99.

https://doi.org/10.4236/ijmpcero.2018.71008
\end{abstract}

Received: December 5, 2017

Accepted: February 24, 2018

Published: February 27, 2018

Copyright $\odot 2018$ by authors and Scientific Research Publishing Inc. This work is licensed under the Creative Commons Attribution International License (CC BY 4.0).

http://creativecommons.org/licenses/by/4.0/ (c) (i) Open Access

\begin{abstract}
This study aimed to determine variations in tomotherapy beam outputs at multiple institutions. Measurements were obtained at 22 radiotherapy institutions. The first parameter was the absolute dose to water $\left(D_{\mathrm{w}, \mathrm{Q}_{\mathrm{msr}}}^{f_{\mathrm{ms}}}\right)$ in the machine-specific reference field ( $f_{\text {msr }}$ ), which indicated a static field in the tomotherapy reference conditions defined by the International Atomic Energy Agency (IAEA) study group. The second measured parameter was the difference between the measured and the planed doses in the intensity modulated radiotherapy (IMRT) verification plans, which were created using a solid phantom by the vendor during tomotherapy apparatus installation to adjust the beam output. The IMRT verification plan error at each institution was defined as the systematic error of the beam output; $D_{\mathrm{w}, Q_{\mathrm{msr}}}^{f_{\text {mr }}}$ was subsequently modified. The $D_{\mathrm{w}, Q_{\mathrm{msr}}}^{f_{\mathrm{ms}}}$ values of four institutions with a modified energy fluence per ideal open time (EFIOT) were lower than the values at other institutions. The mean value of all institutions except those four was $0.994 \pm 0.013$ Gy (range: $0.974 \mathrm{~Gy}, 1.017 \mathrm{~Gy}$ ). When the $D_{\mathrm{w}, Q_{\mathrm{msr}}}^{f_{\text {msr }}}$ value was corrected by the IMRT verification error, this variation decreased. In addition, the mean IMRT verification errors in the TomoDirect ${ }^{\mathrm{TM}}$ and TomoHelical ${ }^{\mathrm{TM}}$ modes with the TomoEDGE ${ }^{\mathrm{TM}}$ mode were $1.2 \% \pm 0.8 \%$ (range: $-0.6 \%, 1.8 \%$ ) and $0.2 \% \pm 0.5 \%$ (range: $-0.6 \%, 0.9 \%)$, respectively $(p<0.05)$. Those without the TomoEDGE mode were $0.6 \% \pm 1.0 \%$ (range: $-1.0 \%, 1.7 \%$ ) and $-0.7 \% \pm 0.7 \%$ (range: $-2.3 \%, 0.4 \%)$, respectively $(p<0.001)$. The variations in tomotherapy beam
\end{abstract}


output across multiple facilities were very low, except at facilities where the EFIOT was modified. The beam output was affected by the mode (e.g., TomoHelical), and it would be recommended to investigate the difference of the beam output due to differences in the mode in order to equalize the beam output.

\section{Keywords}

Tomotherapy, Machine-Specific Reference Field, Multiple Institutions, Beam Output

\section{Introduction}

Tomotherapy (Accuray, Inc., Sunnyvale, CA, USA) is a unique machine for delivering intensity modulated radiation therapy (IMRT) treatments using multileaf collimators (MLCs) of 64 leaves while synchronizing with the gantry rotation and moving the patient into the beam plane at constant speed [1]. However, it is impossible with this machine to measure the absorbed dose to water under standard reference conditions composed of a $10 \mathrm{~cm} \times 10 \mathrm{~cm}$ square field and 100 $\mathrm{cm}$ source-to-surface distance (SSD) or source-to-axis distance (SAD). Therefore, the International Atomic Energy Agency (IAEA) study group defined new reference conditions for treatment units, such as tomotherapy and CyberKnife ${ }^{\mathrm{TM}}$ (Accuray Inc., Sunnyvale, CA, USA), that cannot establish standard reference conditions [2]. The tomotherapy reference conditions were defined as a $5 \mathrm{~cm} \times$ $10 \mathrm{~cm}$ field and $85 \mathrm{~cm} \mathrm{SSD} \mathrm{or} \mathrm{SAD.}$

So far, many national and international dosimetry audits have been reported [3]-[14]. However, there is no large-scale investigation that focused on tomotherapy beam outputs, and the variation is not clear. During multi-institution clinical trials, unification of the doses delivered to tumors and organs-at-risk is an important step toward reducing the number of cases needed for clinical trials and the reliability of the final trial results. The present study aimed to determine variations in these beam outputs among multiple institutions, based on the tomotherapy reference conditions. As tomotherapy delivery synchronizes with the gantry rotation and the couch movement, it is also important to verify the error of IMRT verification plan. Therefore, in this report, it also aimed to clarify the effect of errors due to delivery mode by investigating errors among multiple institutions. The compared delivery modes were TomoHelical ${ }^{\mathrm{TM}}$, TomoDirect ${ }^{\mathrm{TM}}$, and TomoEDGE ${ }^{\mathrm{TM}}$ modes.

\section{Methods}

\subsection{Study Description}

Measurements were obtained at 22 radiotherapy institutions between September 2015 and October 2015. In contrast to the visit or postal audits conducted in the 
context of a multi-institution clinical trial, the radiotherapist at each institution performed measurements using their own equipment, which included a water tank and ionization chamber. Two parameters were measured at each institution. The first parameter was the absolute dose to water $\left(D_{\mathrm{w}, Q_{\mathrm{msr}}}^{f_{\mathrm{msr}}}\right)$ in the machine-specific reference field $\left(f_{\mathrm{msr}}\right)$, which indicated the static field according to the tomotherapy reference conditions defined by the IAEA study group [2]. This parameter was measured to evaluate variations in beam output. The second parameter was the difference between the measured and the planned doses in measurement of the IMRT verification plans, which were created using a cylindrical Virtual Water ${ }^{\text {rx }}$ phantom called "Cheese phantom" (Figure 1(a)) by the vendor during installation of the tomotherapy apparatus to adjust the beam output [15]. The tomotherapy beam output was adjusted to match the calculated values of the TomoTherapy Planning Station ${ }^{\mathrm{TM}}$ (PS, Accuray Inc., Sunnyvale, CA, USA). The IMRT verification plan error at each institution was defined as the residual error of the beam output; subsequently, the absolute dose to water in $f_{\text {msr }}$ was modified.

The measured dataset was provided by the radiotherapist at each institution and included the correction factors of the ionization chamber used for the measurements. We obtained permission for the public release of data from all institutions after masking the institution names.

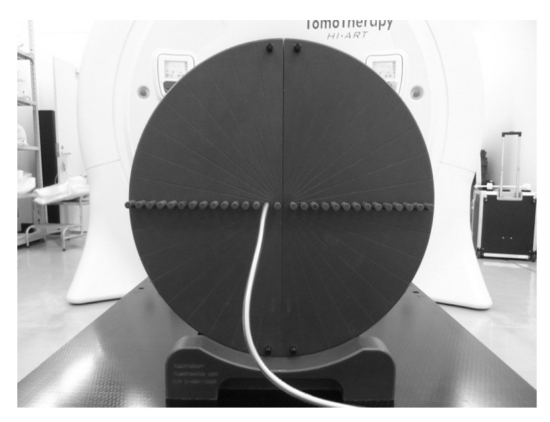

(a)

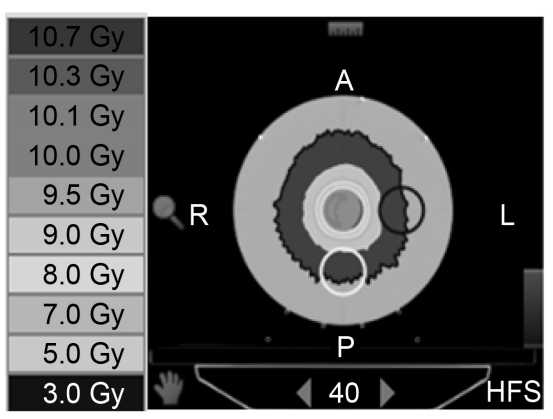

(c)

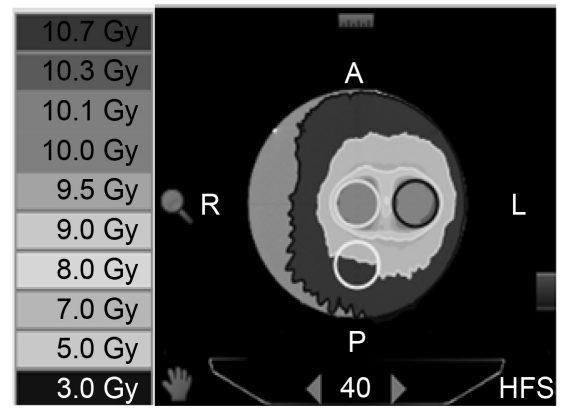

(b)

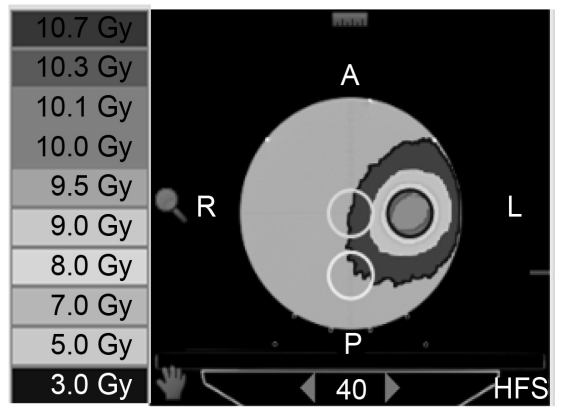

(d)

Figure 1. A photograph of the cylindrical Virtual Water ${ }^{\mathrm{Tw}}$ phantom and representative plans for IMRT verification. (a) The Exradin A1SL ${ }^{\mathrm{TM}}$ ionization chamber can be inserted into a hole in the phantom for dosimetry. (b) Dual target plan: targets were set for central and left regions of interest (ROIs). (c) On-axis plan: a target was set for the central ROI. (d) Off-axis plan: a target was set for the left ROI. 


\subsection{Correction Factors of the Ionization Chambers Used for the Measurements}

All institutions used an Exradin A1SL ionization chamber at an operation voltage of $+300 \mathrm{~V}$, which was supplied by a TomoElectrometer ${ }^{\mathrm{TM}}$ (Standard Imaging, Middleton, WI, USA). The A1SL ionization chambers in all institutions were calibrated by Association for Nuclear Technology in Medicine (ANTM), which is a secondary standard dosimetry laboratory in Japan. The following parameters were obtained from all institutions: dose to water calibration coefficient $\left(N_{\mathrm{D}, \mathrm{W}, \mathrm{Q}_{0}}\right)$, ion recombination correction factor $\left(k_{\mathrm{s}}\right)$, polarity effect correction factor $\left(k_{\mathrm{pol}}\right)$, temperature and pressure correction factor $\left(k_{\mathrm{TP}}\right)$, tissue phantom ratios at depths of 20 and $10 \mathrm{~cm}$ under the tomotherapy reference conditions $\left({ }^{\mathrm{HT}} T P R_{20,10}\right)$, and electrometer calibration factor $\left(k_{\text {elec }}\right)$.

\subsection{Measurement of the Absolute Dose to Water in the Machine-Specific Reference Field; $f_{\mathrm{msr}}$}

The same procedure file was installed in the TomoTherapy Operator Station ${ }^{\text {TM }}$ (OS, Accuray Inc., Sunnyvale, CA, USA) of all cooperating institutions. It consists of a field size of $5 \mathrm{~cm} \times 10 \mathrm{~cm}$, static mode gantry and couch, and a total delivery time of $32 \mathrm{~s}$ (including $20 \mathrm{~s}$ with all binary MLCs closed). Using this installed procedure, absorbed dose measurements were performed for the beam axis at a $10 \mathrm{~cm}$ depth in a water tank with an $85 \mathrm{~cm}$ SSD setup. The absolute dose to water in $f_{\mathrm{msr}}\left(D_{\mathrm{w}, Q_{\mathrm{msr}}}^{f_{\mathrm{mrr}}}\right)$ was defined as follows:

$$
D_{\mathrm{w}, Q_{\mathrm{msr}}}^{f_{\mathrm{mr}}}=M_{Q_{\mathrm{msr}}}^{f_{\mathrm{msr}}} N_{\mathrm{D}, \mathrm{w}, Q_{0}} k_{Q, Q_{0}} k_{Q_{\mathrm{ms}}, Q}^{f_{\text {msr }}, f_{\text {ref }}}
$$

where $M_{Q_{\text {msr }}}^{f_{\text {ms }}}$ is the electrometer reading value in $f_{\text {msr }}$ multiplied by the correction factors $\left(k_{\mathrm{s}}, k_{\mathrm{pol}}, k_{\mathrm{TP}}\right.$ and $\left.k_{\text {elec }}\right), N_{\mathrm{D}, \mathrm{w}, Q_{0}}$ is the dose to water calibration coefficient for the reference beam quality $\left(Q_{0}\right), k_{Q, Q_{0}}$ is the quality conversion factor from $Q_{0}$ to the beam quality $(Q)$ of the standard (conventional) reference conditions $\left(f_{\text {ref }}\right)$, and $k_{Q_{\text {msr }}, Q}^{f_{\text {mst }}, f_{\text {ref }}}$ is the quality conversion factor from $Q$ to the beam quality of the tomotherapy reference conditions $\left(Q_{\mathrm{msr}}\right)$. To calculate $k_{Q, Q_{0}}$, the value that multiplied 1.027 [16] by the ${ }^{\mathrm{HT}} T P R_{20,10}$, was defined as the beam quality index $\left(T P R_{20,10}\right)$ in the conventional reference conditions.

The ANTM provides an estimated relative uncertainty of approximately $0.52 \%$ (coverage factor: 1 ) for the value of $N_{\mathrm{D}, \mathrm{w}, Q_{0}}$. The relative uncertainty regarding the long-term stability of a user's dosimeter, establishment of reference conditions, electrometer reading value, and correction factors were given uncertainties of $0.3 \%, 0.4 \%, 0.6 \%$, and $0.4 \%$ (coverage factor: 1 ), respectively. These uncertainties were proportional to those in the IAEA technical reports series No. 398 [17]. The relative uncertainty for $k_{Q, Q_{0}}$ was assigned a value of $1.0 \%$ for the gamma ray of ${ }^{60} \mathrm{Co}$ [18]. The value of $k_{Q_{\text {msr }}, Q}^{f_{\text {ms }}} f_{\text {ref }}$ was given a value of 1.000 and uncertainty $0.3 \%$ [18]. Measurement expanded the estimated uncertainty by approximately $2.9 \%$ (coverage factor: 2 ).

\subsection{IMRT Verification}

An example of the plans is shown in Figure 1. In Japan, the vendor created a 
dual-target plan (Figure 1(b)) for institutions that installed tomotherapy equipment after July 2013. The vendor created both on-axis (Figure 1(c)) and off-axis plans (Figure 1(d)) for institutions that installed equipment before July 2013. We selected the plan for a jaw width of $5 \mathrm{~cm}$ to match the $f_{\mathrm{msr}}$ from all commissioned jaw widths. In each cooperating institution, the Exradin A1SL ionization chamber was used to perform measurements of each target region. We calculated the mean errors of measurements in the target regions of a dual-target plan or both on-axis and off-axis plans. If an optional license was available for TomoDirect ${ }^{\mathrm{TM}}$ mode and/or TomoEDGE ${ }^{\mathrm{TM}}$ mode, as well as for traditional TomoHelical $^{\mathrm{TM}}$ mode, this institution could provide all error values in the available combination modes. The TomoDirect mode uses a fixed gantry angle instead of rotational beam delivery [19]. The TomoEDGE mode uses dynamic jaw technology with dynamic field width adaptation at the cranial and caudal edges of a target [20]. Either the TomoDirect or TomoHelical mode can be selected for a single plan. Further, the TomoEDGE mode can be included or omitted in each plan. Therefore, institutions with licenses for both the TomoDirect and TomoEDGE modes provided four plans.

Not all institutions used IMRT verification during routine quality assurance/quality control (QA/QC); accordingly, we did not force data provision. Therefore, IMRT verification data were limited.

\subsection{Data Analysis}

The correlation between $D_{\mathrm{w}, Q_{\mathrm{msr}}}^{f_{\text {mr }}}$ and IMRT verification error was assessed using the Spearman's rank correlation $(r)$. In order to know the true variation of machine output, we revised $D_{\mathrm{w}, Q_{\mathrm{msr}}}^{f_{\text {ms }}}$ according to the IMRT verification plan error using the following equation:

$$
{ }^{C} D_{\mathrm{w}, Q_{\mathrm{msr}}}^{f_{\mathrm{msr}}}=D_{\mathrm{w}, Q_{\mathrm{msr}}}^{f_{\mathrm{msr}}}(1-E / 100)
$$

where, ${ }^{C} D_{\mathrm{w}, Q_{\text {msr }}}^{f_{\text {msr }}}$ is the corrected $D_{\mathrm{w}, Q_{\mathrm{msr}}}^{f_{\text {msr }}}$ and $E$ represents the percentage IMRT verification plan error.

In addition, Wilcoxon signed rank test was used to evaluate and compare the systematic IMRT verification errors of the TomoDirect and TomoHelical modes.

$\mathrm{R}^{\mathrm{TM}}$ statistical software (version 2.15.2; R Project for Statistical Computing; Vienna, Austria) was used for all analyses. P values $<0.05$ were considered to indicate significance.

\section{Results}

\subsection{Variations in Parameters of the Exradin A1SL Ionization Chamber}

Figure 2 shows the variations of $N_{\mathrm{D}, \mathrm{W}, Q_{0}}, k_{\mathrm{s}}$ and $k_{\mathrm{pol}}$ of the Exradin A1SL ionization chambers used by the participating institutions. The mean values of $N_{\mathrm{D}, \mathrm{W}, \mathrm{Q}_{0}}, k_{\mathrm{s}}$ and $k_{\mathrm{pol}}$ were $0.570 \pm 0.014 \mathrm{~Gy} / \mathrm{nC}$ (range: $0.534 \mathrm{~Gy} / \mathrm{nC}, 0.594$ $\mathrm{Gy} / \mathrm{nC}$ ), $1.003 \pm 0.001$ (range: $1.000,1.005$ ), and $1.000 \pm 0.002$ (range: 0.999, $1.007)$, respectively. One institution reported an outlying high $k_{\text {pol }}$ value of 1.007 . 


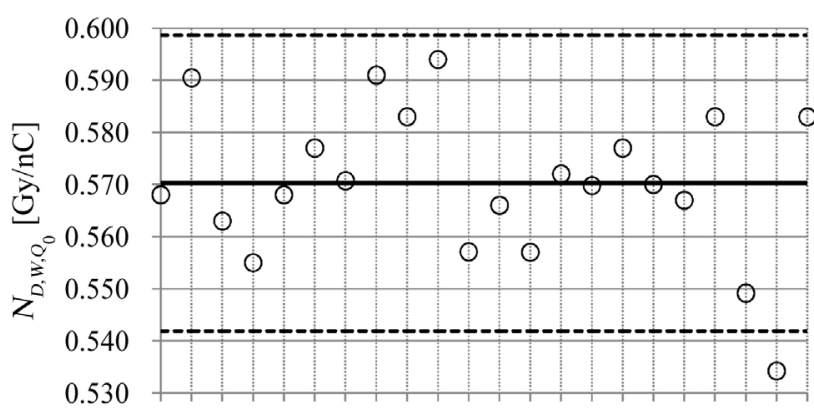

12345678910111213141516171819202122

Center number

(a)

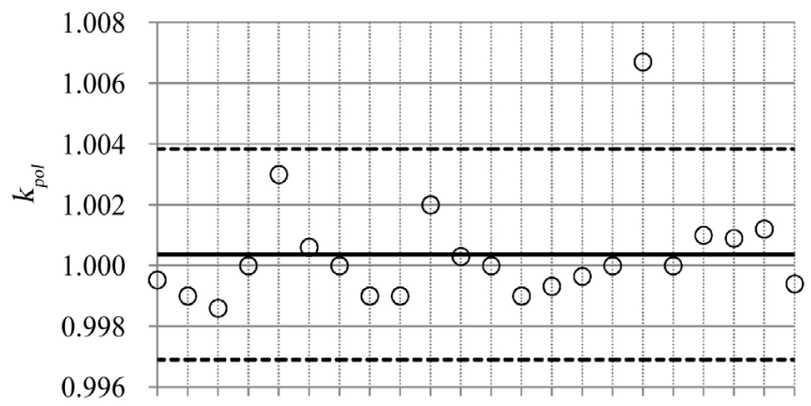

12345678910111213141516171819202122

Center number

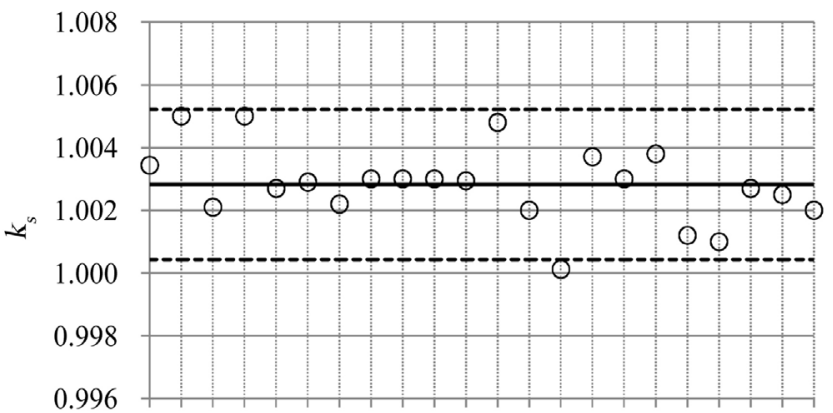

12345678910111213141516171819202122

Center number

(b)

(c)

Figure 2. Variations in parameters of the Exradin A1SL ionization chambers. (a) Dose to water calibration coefficient, $N_{\mathrm{D}, \mathrm{W}}$. (b) Recombination correction factor, $k_{s}$ (c) Polarity correction factor, $k_{\text {pol }}$. A solid line on each figure shows mean value, and two dashed lines show $95 \%$ confidence intervals.

Among the other parameters, the $k_{\text {elec }}$ values were 1.000 in many institutions, except for one institution that reported a value of 0.997. Although the ${ }^{\mathrm{HT}} T P R_{20,10}$ values varied widely from 0.606 to 0.627 , the $k_{Q, Q_{0}}$ value in this range was 1.001 .

\subsection{Absolute Dose to Water in the Machine-Specific Reference Field; $f_{\mathrm{msr}}$}

Figure 3 shows the values of $D_{\mathrm{w}, Q_{\mathrm{msr}}}^{f_{\mathrm{ms}}}$. Four institutions had lower values relative to the other institutions. The mean value of the latter (excluding the four institutions) was $0.994 \pm 0.013 \mathrm{~Gy}$ (range: $0.974 \mathrm{~Gy}, 1.017 \mathrm{~Gy}$ ).

\subsection{IMRT Verification}

Measured data were provided from 22 institutions. Table 1 shows the IMRT verification modality used by the institutions involved in this study. Data of four institutions with low $D_{\mathrm{w}, Q_{\mathrm{msr}}}^{f_{\text {ms }}}$ values were not used for analysis.

Figure 4 shows the scatter plots of the $D_{\mathrm{w}, Q_{\mathrm{msr}}}^{f_{\text {mr }}}$ values versus the mean percentage errors in the IMRT verification measurements. The correlation coefficients of plans using the TomoDirect mode without (Figure 4(a)) and with the 


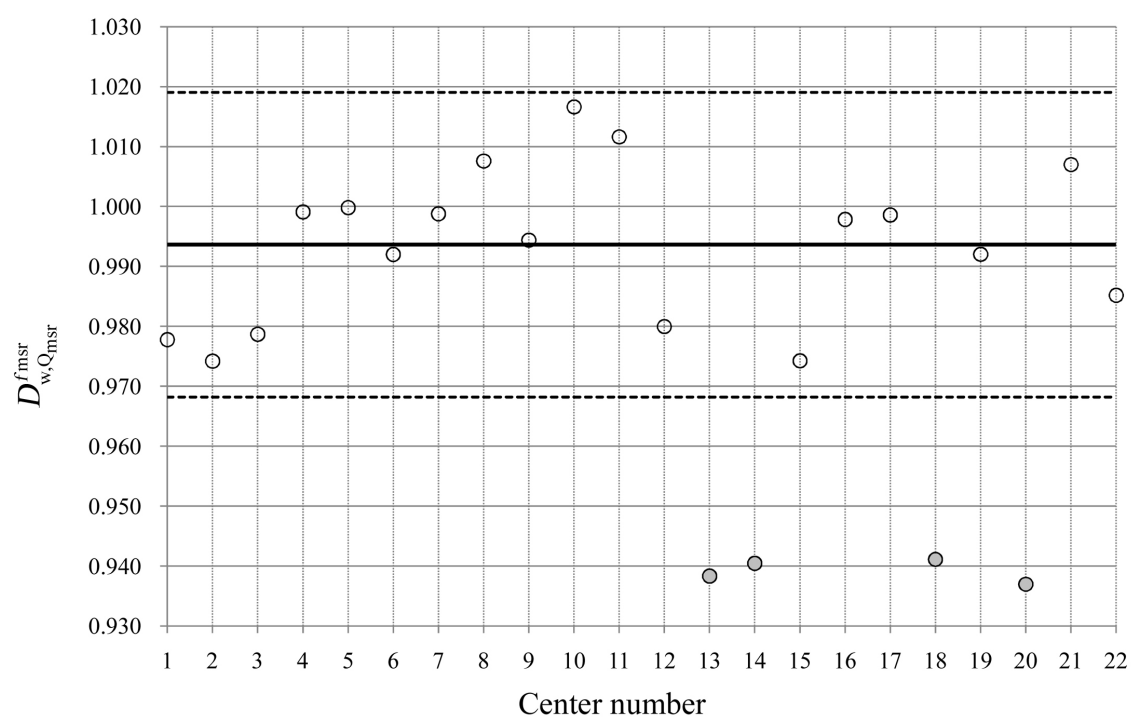

Figure 3. Variations in the absolute doses to water in machine-specific reference fields at multiple institutions. Four institutions (circles filled in grey color) had lower values relative to the other institutions. Excluding these four data, the solid line indicates the mean value of the corrected values, and the two dashed lines indicate $95 \%$ confidence intervals.

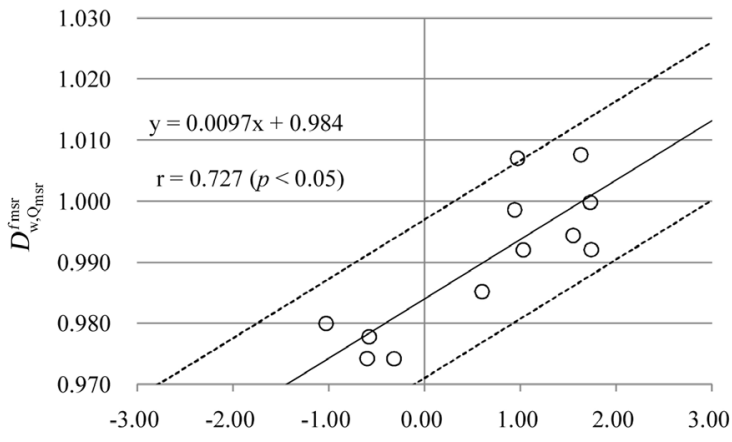

The mean percentage IMRT verification error [\%]

(a)

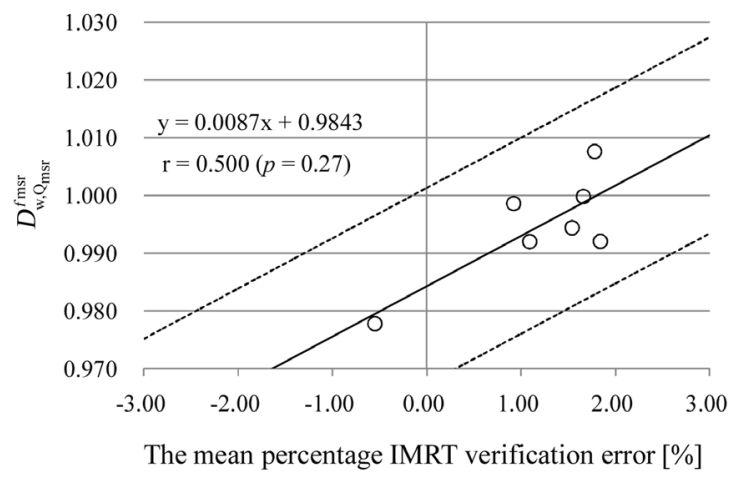

(b)

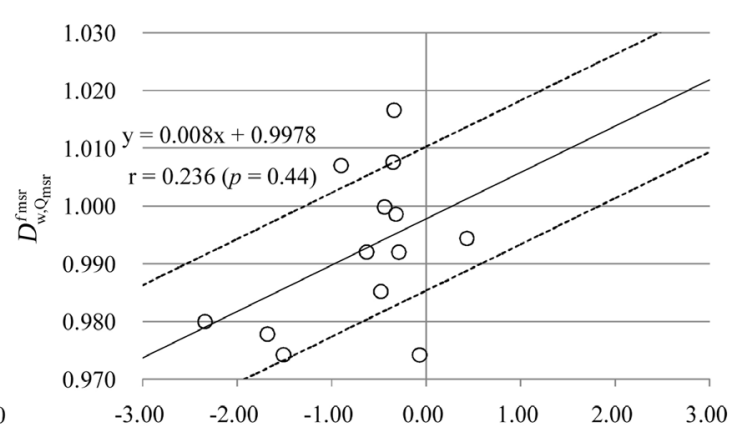

The mean percentage IMRT verification error [\%]

(c)

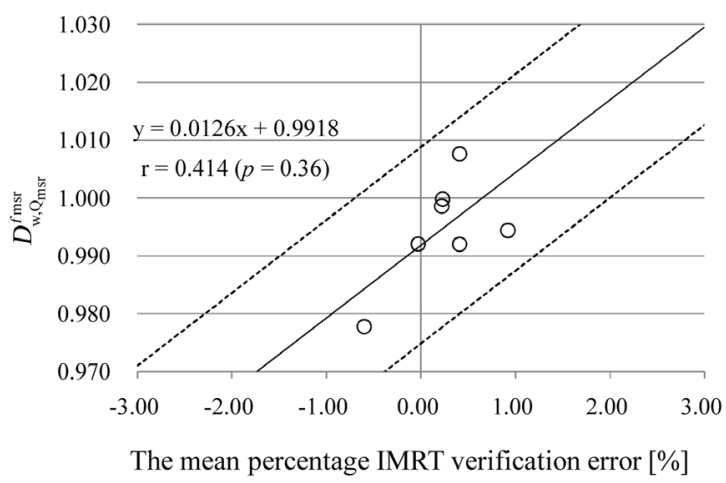

(d)

Figure 4. Correlation between the mean percentage IMRT verification error and absolute dose to water in a machine-specific reference field. Correlation coefficients for the combinations of (a) TomoDirect without TomoEDGE, (b) TomoDirect with TomoEDGE, (c) TomoHelical without TomoEDGE, and (d) TomoHelical with TomoEDGE; the respective values were 0.727 (Spearman's rank correlation, $p<0.05), 0.500(p=0.27), 0.236(p=$ $0.44)$, and $0.414(p=0.36)$. Two dashed lines on each figure show $95 \%$ prediction intervals. 
Table 1. The number of IMRT verification modality used by the institutions involved in this study.

\begin{tabular}{ccc}
\hline & TomoDirect & TomoHelical \\
\hline With TomoEdge & 7 & 7 \\
Without TomoEdge & 12 & 13
\end{tabular}

TomoEDGE modes (Figure 4(b)) were 0.727 (Spearman's rank correlation, $p<$ $0.05)$ and $0.500(p=0.27)$, respectively. The correlation coefficients of plans using the TomoHelical mode without (Figure $4(c)$ ) and with the TomoEDGE modes (Figure $4(\mathrm{~d}))$ were $0.236(p=0.44)$ and $0.414(p=0.36)$, respectively.

We corrected the $D_{\mathrm{w}, Q_{\mathrm{msr}}}^{f_{\mathrm{mr}}}$ values using Equation (2) and the error from each institution that used the TomoDirect mode without the TomoEDGE mode. Figure 5 shows the values of ${ }^{C} D_{\mathrm{w}, Q_{\text {msr }}}^{f_{\text {ms }}}$ and $D_{\mathrm{w}, Q_{\text {msr }}}^{f_{\text {msr }}}$. The mean value of ${ }^{C} D_{\mathrm{w}, Q_{\text {msr }}}^{f_{\text {msr }}}$ was $0.984 \pm 0.007 \mathrm{~Gy}$ (range: $0.975 \mathrm{~Gy}, 0.997 \mathrm{~Gy}$ ). The variation of ${ }^{C} D_{\mathrm{w}, Q_{\text {mar }}}^{f_{\text {ms }}}$ decreased to a greater extent than that of $D_{\mathrm{w}, Q_{\mathrm{msr}}}^{f_{\mathrm{mrr}}}$. Similarly, the values of ${ }^{C} D_{\mathrm{w}, Q_{\mathrm{msr}}}^{f_{\mathrm{msr}}}$ obtained using TomoDirect with the TomoEDGE mode, TomoHelical with the TomoEDGE mode, and TomoHelical without the TomoEDGE mode were $0.980 \pm$ $0.011 \mathrm{~Gy}$ (range: $0.974 \mathrm{~Gy}, 0.990 \mathrm{~Gy}$ ), $0.989 \pm 0.011 \mathrm{~Gy}$ (range: $0.984 \mathrm{~Gy}, 1.003$ Gy), and $0.998 \pm 0.011 \mathrm{~Gy}$ (range: $0.950 \mathrm{~Gy}, 1.020 \mathrm{~Gy}$ ), respectively.

Figure 6 shows the errors between calculated and measured IMRT verification values. The mean IMRT verification errors in the TomoDirect and TomoHelical modes with the TomoEDGE mode were $1.2 \% \pm 0.8 \%$ (range: $-0.6 \%$, $1.8 \%$ ) and $0.2 \% \pm 0.5 \%$ (range: $-0.6 \%, 0.9 \%$ ), respectively (Wilcoxon signed rank test, $p<0.05)$. The mean IMRT verification errors in the TomoDirect and TomoHelical modes without the TomoEDGE mode were $0.6 \% \pm 1.0 \%$ (range: $-1.0 \%, 1.7 \%)$ and $-0.7 \% \pm 0.7 \%$ (range: $-2.3 \%, 0.4 \%)$, respectively $(p<0.001)$.

\section{Discussion}

The Exradin A1SL ionization chamber was used for measurement in this investigation, which was routinely used for all institutions. Therefore, the data shown in Figure 2 would be useful for determining whether the A1SL ionization chamber of each institution is functioning normally. Muir at al. reported that the standard uncertainty of the normalized $N_{\mathrm{D}, \mathrm{W}, \mathrm{Q} 0}$ coefficients among the chambers was $3.4 \%$ [21]. According to our results, the standard uncertainty of the normalized $N_{\mathrm{D}, \mathrm{W}, \mathrm{Q} 0}$ coefficients was $2.5 \%$, determined as the standard deviation $(=0.014 \mathrm{~Gy} / \mathrm{nC})$ divided by the mean value $(=0.570 \mathrm{~Gy} / \mathrm{nC})$. The variation in $N_{\mathrm{D}, \mathrm{W}, \mathrm{Q} 0}$ coefficients in this study was comparable to that in previous reports. Regarding $k_{\text {pol }}$, the American Association of Physicists in Medicine (AAPM) Report 67, addendum to task group (TG) 51 protocol, recommended that the variation in polarity correction factors from unity should remain within $\pm 0.4 \%$ at any energy [22]. Only one institution had a polarity correction factor with a difference of $+0.7 \%$ from unity. Although $k_{\text {pol }}$ was remeasured in the chamber at that institution, the value did not change. A major leakage current was also not 


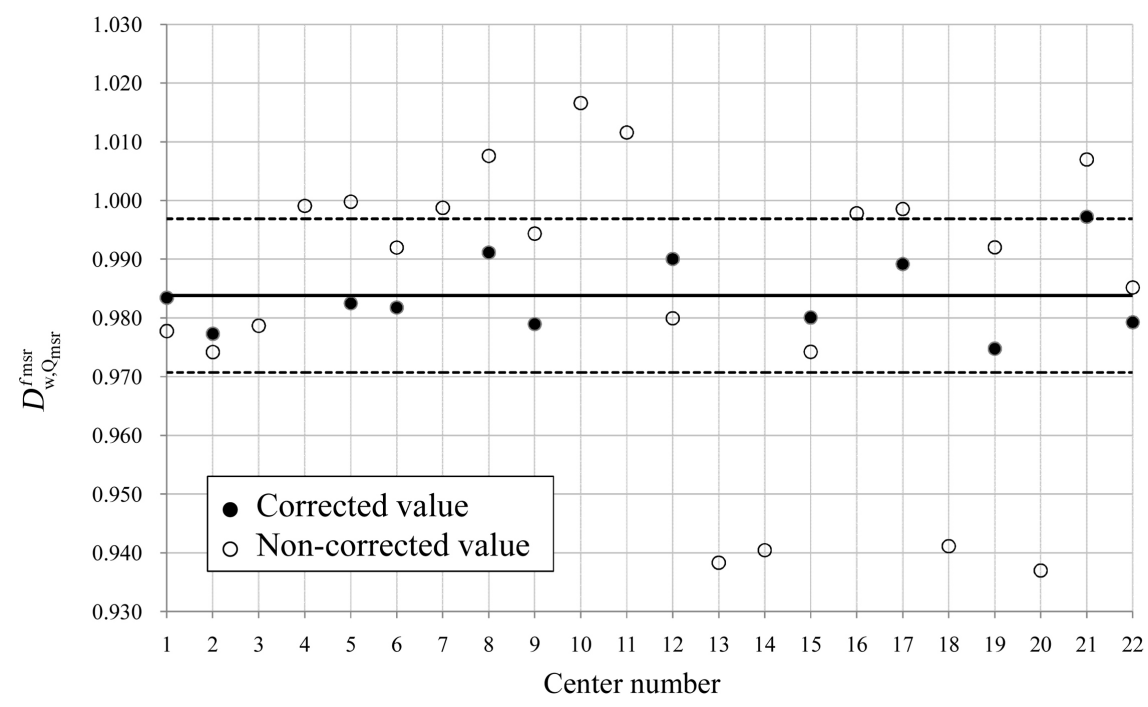

Figure 5. Variations in the corrected and non-corrected absolute doses to water in the machine-specific reference fields at multiple institutions. The solid line indicates the mean value of the corrected values, and the two dashed lines indicate $95 \%$ confidence intervals.

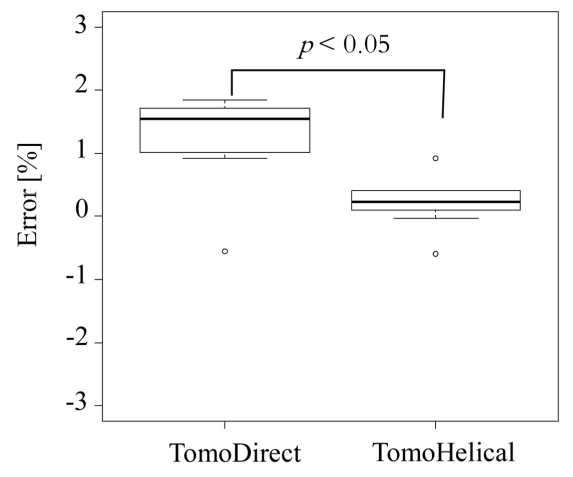

(a)

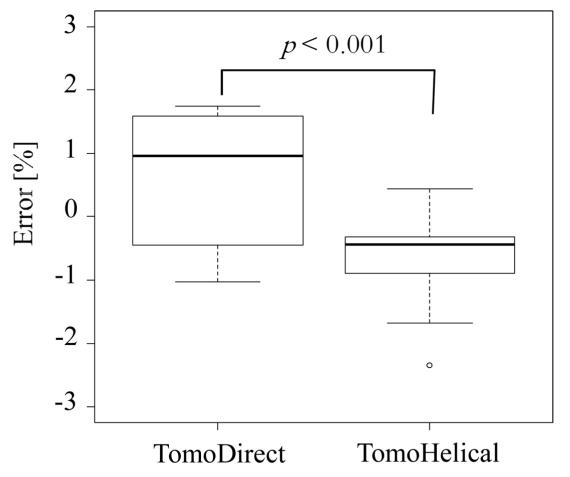

(b)

Figure 6. The error between the calculated and measured IMRT verification values (a) with and (b) without the TomoEDGE mode; these errors differed significantly between the TomoDirect and TomoHelical modes (Wilcoxon signed rank test, $p<0.05$ with the TomoEDGE mode and $p<0.001$ without the TomoEDGE mode).

detected. Because all other institutions had a $k_{\text {pol }}$ value of 1.000 , the effect of the uncertainty of $k_{\mathrm{pol}}$ on $D_{\mathrm{w}, Q_{\mathrm{msr}}}^{f_{\text {msr }}}$ was small.

Ost et al. reported that the mean static output of the tomotherapy equipment at four institutions was $6.238 \pm 0.058 \mathrm{~Gy}$ (one standard deviation; 1 SD) for $60 \mathrm{~s}$ at a depth of $10 \mathrm{~cm}$ in water and $85 \mathrm{~cm} \mathrm{SAD} \mathrm{[23].} \mathrm{In} \mathrm{our} \mathrm{results,} \mathrm{the} \mathrm{mean} \mathrm{value}$ of the outputs at multiple institutions was $0.994 \pm 0.013 \mathrm{~Gy}(1 \mathrm{SD})$ for $12 \mathrm{~s}$ at a depth of $10 \mathrm{~cm}$ in water and $85 \mathrm{~cm}$ SSD. If the delivery time and measurement distance are revised via calculations, our result yield $6.208[=0.994 \times(60 / 12) \times$ $\left.(95 / 85)^{2}\right]$. In fact, our results included a few binary MLC transmission (approximately $0.3 \%$ [24]), but our results did not demonstrate a significant difference in output compared to previous reports. In this investigation, the expanded un- 
certainty (coverage factor: 2$)$ could be estimated as $2.6 \%(=2 \times 100 \times 0.013 / 0.994)$ from the actual measurement value. This value was approximately equivalent to the expected value (2.9\%), as described above. The variation in beam output was adequately small and within expectable uncertainty.

The $D_{\mathrm{w}, Q_{\mathrm{msr}}}^{f_{\text {msr }}}$ of the four institutions was lower than that of the others (Figure 3). A modeling parameter, energy fluence per ideal open time (EFIOT), is used in tomotherapy beam models [25] [26]. EFIOT defines the tomotherapy dose rate in the PS and is usually given as a specific value at all institutions. However, in Japan, some early adopters of tomotherapy requested decreases in their institutional EFIOT values because of legal limitations (the upper limit of dose rate). Therefore, it was necessary for these institutions to achieve decreases in tomotherapy beam outputs that would equal the calculated value of the PS. Through an interview survey, we determined that the four institutions had intentionally decreased the EFIOT values.

The $D_{\mathrm{w}, Q_{\mathrm{msr}}}^{f_{\text {ssr }}}$ value showed the strongest correlation with the mean percentage IMRT verification error for combinations of the TomoDirect mode without the TomoEDGE mode in all combinations of the mode (Figure 4(a)). The reason for this strong correlation would be that the fixed gantry and the fixed jaws were used in this delivery mode. In addition, the error of the TomoHelical mode (Figure 4(c) and Figure 4(d)) was smaller than that of the TomoDirect (Figure 4(a) and Figure 4(b)). This result demonstrates that the frequency of use of the TomoHelical mode is clinically high in many institutions and that the output is adjusted so that the error of this mode is small.

When the $D_{\mathrm{w}, Q_{\text {ms }}}^{f_{\text {ms }}}$ value was corrected by the measured IMRT verification error, the variation in this value decreased as shown in Figure 5. This result demonstrates that appropriate adjustment of the beam output at each institution would lead to further decreases in inter-institutional variations in $D_{\mathrm{w}, Q_{\mathrm{msr}}}^{f_{\text {mr }}}$. It is difficult to achieve complete accord of the measured IMRT verification errors among all commissioned jaw widths. Therefore, the beam output is adjusted to minimize the mean error at all commissioned jaw widths or at a high-priority jaw width. However, the beam modeling parameter jaw fluence output factor (JFOF) allows adjustment to accommodate differences in fluence at each jaw width [25] [26]. By precisely adjusting the JFOF value for each jaw width, the beam output of each institution will be able to achieve unity.

To the best of our knowledge, this is the first report to describe differences in errors between measurements obtained in the TomoDirect and TomoHelical modes. Although Schiefer et al. reported the results of a TLD audit for the TomoHelical and the TomoDirect modes, differences in errors between these modes were not described in the report [3]. The current commissioning process does not include a beam modeling parameter to correct differences in the errors between measurements according to mode. In the future, this difference might be canceled by the introduction of a new beam modeling parameter in the PS, such as a parameter that would allow adjustment of the fluence in each mode. Modification of the fluence per mode would allow the beam output to equalize. 
However, as the cause of the difference is not clear at present, so it is necessary to investigate the difference as the next approach.

This study has one notable limitation. This investigation did not comprise a visit or postal audit. Because one radiotherapist in every institution performed measurements using their own equipment, the measurement processes may have introduced some operator-dependent uncertainty into the results. We are planning to visit or postal measurements to grasp the uncertainty in detail.

\section{Conclusion}

We investigated the tomotherapy beam outputs at 22 institutions using an Exradin A1SL ionization chamber at each institution. For the chamber correction factors (e.g., $N_{\mathrm{D}, \mathrm{W}, Q_{0}}, k_{\mathrm{s}}, k_{\mathrm{pol}}$ ), we provided the standard values in the presented data. The presented data are therefore beneficial information for tomotherapy users. In addition, the value of $D_{\mathrm{w}, \mathrm{Q}_{\text {msr }}}^{f_{\text {ms }}}$ was similar to that of a previously published paper. The variation in $D_{\mathrm{w}, \mathrm{Q}_{\mathrm{msr}}}^{f_{\text {ms }}}$ was small, except at institutions where EFIOT had been modified, and remained within the uncertainty. Currently, modes (e.g., TomoHelical) affect beam output; therefore, it would be recommended to investigate the mode dependent beam output difference in order to equalize the beam output.

\section{Acknowledgements}

We are grateful to the staff at all cooperating institutions. The authors would like to thank Enago (www.enago.jp) for the English language review.

\section{References}

[1] Mackie, T.R. (2016) History of Tomotherapy. Physics in Medicine \& Biology, 51, R427-R453. https://doi.org/10.1088/0031-9155/51/13/R24

[2] Alfonso, R., Andreo, P., Capote, R., Huq, M.S., Kilby, W., Kjäll, P., Mackie, T.R., Palmans, H., Rosser, K., Seuntjens, J., Ullrich, W. and Vatnitsky, S. (2008) A New Formalism for Reference Dosimetry of Small and Nonstandard Fields. Medical Physics, 35, 5179-5186. https://doi.org/10.1118/1.3005481

[3] Schiefer, H., Buchauer, K., Heinze, S., Henke, G. and Plasswilm, L. (2015) Design and Implementation of a "Cheese" Phantom-Based Tomotherapy TLD Dose Intercomparison. Strahlentherapie und Onkologie, 191, 855-861.

https://doi.org/10.1007/s00066-015-0850-2

[4] Izewska, J., Svensson, H. and Ibbott, G. (2002) Worldwide Quality Assurance Networks for Radiotherapy Dosimetry. Standards and Codes of Practice in Medical Radiation Dosimetry. Proceedings of an International Symposium, 2, International Atomic Energy Agency, Vienna.

[5] Izewska, J., Georg, D., Bera, P., Thwaites, D., Arib, M., Saravi, M., Sergieva, K., Li, K., Yip, F.G., Mahant, A.K. and Bulski, W. (2007) A Methodology for TLD Postal Dosimetry Audit of High-Energy Radiotherapy Photon Beams in Non-Reference Conditions. Radiotherapy and Oncology, 84, 67-74. https://doi.org/10.1016/j.radonc.2007.06.006

[6] Ebert, M.A., Howlett, S.J., Harrison, K., Cornes, D., Hamilton, C.S. and Denham, J.D. (2008) Linear-Accelerator X-Ray Output: A Multicentre Chamber-Based In- 
tercomparison Study in Australia and New Zealand. Australasian Physical and Engineering Science in Medicine, 31, 268-279. https://doi.org/10.1007/BF03178596

[7] Kim, J.I., Chung, J.B., Park, Y.K., Song, J.Y., Kim, S.K., Ahn, S.H., Choi, C.H., Choi ,W.H., Cho, B., Ju, S.G., Kim, S.J. and Ye, S.J. (2013) A Multi-Institutional Study for Tolerance and Action Levels of IMRT Dose Quality assurance Measurements in Korea. Journal of Applied Clinical Medical Physics, 14, 3964. https://doi.org/10.1120/jacmp.v14i2.3964

[8] Ferreira, I.H., Dutreix, A., Bridier, A., Chavaudra, J. and Svensson, H. (2000) The ESTRO-Quality Assurance Network (EQUAL). Radiotherapy and Oncology, 55, 273-284. https://doi.org/10.1016/S0167-8140(99)00101-2

[9] Gillis, S., De Wagter, C., Bohsung, J., Perrin, B., Williams, P. and Mijnheer, B.J. (2005) An Inter-Centre Quality Assurance Network for IMRT Verification: Results of the ESTRO QUASIMODO Project. Radiotherapy and Oncology, 76, 340-353. https://doi.org/10.1016/j.radonc.2005.06.021

[10] Lye, J., Kenny, J., Lehmann, J., Dunn, L., Kron, T., Alves, A., Cole, A. and Williams, I. (2014) A 2D Ion Chamber Array Audit of Wedged and Asymmetric Fields in an Inhomogeneous Lung Phantom. Medical Physics, 41, 101712. https://doi.org/10.1118/1.4896097

[11] Kumar, R., Sharma, S.D., Deshpande, S., Sresty, N.V., Bhatt, C.P., Amols, H.I., Chourasiya, G. and Mayya, Y.S. (2014) Analysis of Patient Specific Dosimetry Quality Assurance Measurements in Intensity Modulated Radiotherapy: A Multi Centre Study. Journal of Cancer Research and Therapeutics, 10, 611-617.

[12] Mizuno, H., Fukumura, A., Fukahori, M., Sakata, S., Yamashita, W., Takase, N., Yajima, K., Katayose, T., Abe-Sakama, K., Kusano, Y., Shimbo, M. and Kanai, T. (2014) Application of a Radiophotoluminescent Glass Dosimeter to Nonreference Condition Dosimetry in the Postal Dose Audit System. Medical Physics, 41, 112104. https://doi.org/10.1118/1.4898596

[13] Budgell, G., Berresford, J., Trainer, M., Bradshaw, E., Sharpe, P. and Williams, P. (2011) A National Dosimetric Audit of IMRT. Radiotherapy and Oncology, 99, 246252. https://doi.org/10.1016/j.radonc.2011.03.016

[14] Clark, C.H., Hansen, V.N., Chantler, H., Edwards, C., James, H.V., Webster, G., Miles, E.A., Guerrero Urbano, M.T., Bhide, S.A., Bidmead, A.M. and Nutting, C.M. (2009) Dosimetry Audit for a Multi-Centre IMRT Head and Neck Trial. Radiotherapy and Oncology, 93, 102-108. https://doi.org/10.1016/j.radonc.2009.04.025

[15] Kinhikar, R.A., Jamema, S.V., Reenadevi, P.R, Zubin, M., Gupta, T., Dhote, D.S., Deshpande, D.D., Shrivastava, S.K. and Sarin, R. (2009) Dosimetric Validation of First Helical Tomotherapy Hi-Art II Machine in India. Journal of Medical Physics, 34, 23-30. https://doi.org/10.4103/0971-6203.48717

[16] Jeraj, R., Mackie, T.R., Balog, J. and Olivera, G. (2005) Dose Calibration of Nonconventional Treatment Systems Applied to Helical Tomotherapy. Medical Physics, 32, 570-577. https://doi.org/10.1118/1.1855015

[17] Andreo, P. (2000) Absorbed Dose Determination in External Beam Radiotherapy: An International Code of Practice for Dosimetry Based on Standards of Absorbed Dose to Water. IAEA Technical Report Series No. 398, International Atomic Energy Agency, Vienna.

[18] Japan Society of Medical Physics (2012) Standard Dosimetry of Absorbed Dose to Water in External Beam Radiotherapy (Standard Dosimetry 12). Tsusho-Sangyo Kenkyu-Sha.

[19] Hashimoyo, H., Omura, M., Matsui, K., Mukai, Y., Hongo, H., Yamakabe, W., Sai- 
to, K. and Yoshida, M. (2015) Tangent Field Technique of TomoDirect Improves Dose Distribution for Whole-Breast Irradiation. Journal of Applied Clinical Medical Physics, 16, 5369.

[20] Sugie, C., Manabe, Y., Hayashi, A., Murai, T., Takaoka, T., Hattori, Y., Iwata, H., Takenaka, R. and Shibamoto, Y. (2015) Efficacy of the Dynamic Jaw Mode in Helical Tomotherapy with Static Ports for Breast Cancer. Technology in Cancer Research \& Treatment, 14, 459-465. https://doi.org/10.1177/1533034614558746

[21] Muir, B.R. (2015) Ion Chamber Absorbed Dose Calibration Coefficients, $N_{D, \text { w }}$ Measured at ADCLs: Distribution Analysis and Stability. Medical Physics, 42, 1546-1554. https://doi.org/10.1118/1.4914381

[22] McEwen, M., DeWerd, L., Ibbott, G., Followill, D., Rogers, D.W., Seltzer, S. and Seuntjens, J. (2014) Addendum to the AAPM's TG-51 Protocol for Clinical Reference Dosimetry of High-Energy Photon Beams. Medical Physics, 41, Article ID: 041501. https://doi.org/10.1118/1.4866223

[23] De Ost, B., Schaeken, B., Vynckier, S., Sterpin, E. and Van den Weyngaert, D. (2011) Reference Dosimetry for Helical Tomotherapy: Practical Implementation and a Multicenter Validation. Medical Physics, 38, 6020-6026.

https://doi.org/10.1118/1.3651496

[24] Kinhikar, R.A. (2008) Multileaf Collimator Transmission from the First Hi-Art II Helical Tomotherapy Machine in India. Journal of Cancer Research and Therapeutics, 4, 88-90. https://doi.org/10.4103/0973-1482.42255

[25] Sandra, C.L. (2011) Dose Optimization in TomoTherapy. EPU-HEGP.

[26] Accuray (2014) 1d-Beam Model and Machine Settings. 